\title{
The Stellar Populations of Seyfert 2 Nuclei
}

\author{
Marc Sarzi ${ }^{1}$, Joseph C. Shields ${ }^{2}$, Richard W. Pogge \\ and Paul Martini ${ }^{3}$
}

${ }^{1}$ Centre for Astrophysics Research, University of Hertfordshire, AL10 9AB Hatfield, UK
${ }^{2}$ Physics \& Astronomy Department, Ohio University, Athens, OH 45701 USA
${ }^{3}$ Department of Astronomy, The Ohio State University, Columbus, OH 43210, USA

\begin{abstract}
We present a preliminary analysis of the stellar populations in the central parsecs of a sample of 22 Seyfert 2 galaxies, based on a careful separation of nebular emission and stellar light in high-spatial resolution $H S T$-STIS spectra. $10 \%$ of the surveyed nuclei display stellar populations of intermediate age, $\sim 1-2$ Gyr old, whereas the remaining targets appear to be evenly split between objects showing only very old stellar populations and nuclei requiring also an additional blue featureless component, which we characterise by means of very young, few-Myr-old stars. The small fraction of stellar population of intermediate age seems to argue against the presence of such a young component, however, since the short lifetime of O-stars would imply recurrent star-formation episodes and the build-up over the last 1-2 Gyr of a detectable intermediate-age population. Additionally, a correlation between the luminosity of such a blue component and the emission from highly-ionised species, together with the general absence of Wolf-Rayet features, further suggests that the featureless continuum arises from the central engine rather than from star-forming regions. We discuss our results in the framework of the unification paradigm and of models for star formation close to supermassive black holes.
\end{abstract}

Keywords. galaxies: nuclei, galaxies: Seyfert, galaxies: stellar content

\section{Introduction}

The study of the stellar population content of galactic nuclei is crucial to our understanding of the AGN phenomenon, as it allows to assess the relative rôle of star formation and accretion onto the central supermassive black hole $(\mathrm{SMBH})$ in powering the observed nebular activity. In the specific case of Seyfert 2 nuclei (Sy2s), constraining the presence of young stars is also relevant to unification theory (Antonucci 1993). While Sy2s might not show a broad-line region (BLR) due to the unfavorable orientation of an obscuring torus, they nonetheless exhibit a featureless blue continuum like that in their Type 1 counterparts. This would appear to be problematic for the unification theory since such a component, which presumably arises from an accretion disk, should also be blocked by the obscuring material. As a possible solution, it has been suggested that the blue continuum of Sy2s originates instead in star forming regions (e.g., Colina et al. 1997; González Delgado et al. 1998). Yet, given the limited spatial resolution characterising most spectroscopic studies of Sy2s, typically subtending a few 100 pc, it is still unclear whether such star formation is truly nuclear.

To address this issue we have carried out an observing campaign of nearby Sy2s with the STIS spectrograph on board the Hubble Space Telescope (HST), in order to constrain the amount of star formation as close as possible to the center.

\section{Sample and Data}

Our sample consists of 19 nearby Seyfert 2 galaxies that were selected from the combined CfA and Revised Shapley-Ames Seyfert samples (Osterbrock \& Martel 1993; 
Mulchaey et al. 1997) to have receding velocities $v<5000 \mathrm{~km} \mathrm{~s}^{-1}$ and a distinct, unobscured nucleus from archival HST images. To this sample we added 3 Seyfert 2 galaxies from the Survey of Nearby Nuclei with STIS (SUNNS, Shields et al. 2007), which in turn were selected from the Palomar spectroscopic survey (Ho et al. 1997) based only on proximity $(D<17 \mathrm{Mpc})$ and a minimum flux of $\mathrm{H} \alpha+[\mathrm{N} \mathrm{II}]$ line emission.

For all these Sy2s, two-dimensional spectra were obtained with the G430L and G750M gratings, yielding spectra spanning $3300-5700$ and $6300-6850 \AA$ with FWHM spectral resolution for extended sources of 10.9 and $2.2 \AA$, respectively. From these data we extracted one-dimensional spectra over a $0^{\prime \prime} .25 \times 0^{\prime \prime} .2$ aperture, corresponding to a circular aperture with a radius $R=0^{\prime \prime} .13$, or $29 \mathrm{pc}$ for the mean sample distance of $\sim 46 \mathrm{Mpc}$.

\section{Method}

In order to place constraints on the star-formation history of our sample Sy2s, we modeled the stellar continuum in the STIS spectra with linear combinations of singleage stellar population models from Bruzual \& Charlot (2003), with weights and velocity broadening optimized using the direct-fitting method described in Sarzi et al. (2006). This is similar to the method used by Sarzi et al. (2005) in the case of the entire SUNNS sample, except in that the spectral regions affected by nebular emission are no longer excluded from the fitting process, since the emission-lines are treated as Gaussian templates and fitted simultaneously with the stellar templates to the observed spectra. This has the advantage of maximising the spectral information available to the fitting algorithm, which is crucial when dealing with Sy2s where most, if not all, of the age- and metallicitysensitive stellar absorption features are heavily contaminated by ionised-gas emission.

In fitting the spectra we also include reddening due to interstellar dust, both in the Milky Way and in the sample galaxies, and due to dust in the emission-line regions. The latter affects only the fluxes of the emission-line templates, and is constrained by the expected decrement of the Balmer lines. In matching the nebular emission we imposed the same kinematics to all forbidden lines, and likewise for the recombination lines. Finally, we allowed for complex line profiles using multiple Gaussian components.

For the stellar population analysis it is critical to constrain the strength of the highorder Balmer emission lines and the exact profiles of the strongest lines. Accounting for their different spectral resolution, we thus fitted both blue and red spectra at the same time, which allows to exploit the longest wavelength leverage and to match also the profiles of the strong $\mathrm{H} \alpha$ and [N II] lines.

\section{Results}

Using stellar population templates of solar metallicity ranging in age from $1 \mathrm{Myr}$ to $10 \mathrm{Gyr}$, we found that $10 \%$ of the surveyed nuclei display stellar populations of intermediate age, 1-2 Gyr old, whereas the remaining Sy2s appear to be evenly split between objects showing only very old stellar populations and nuclei requiring also an additional blue featureless component, which here we characterise by means of very young, fewMyr-old stars. Figure 1 shows two examples of this last class of Sy2s, which are also characterised by strong nebular emission.

Although the presence of a very young population seems required in almost half of the sample, we need to keep in mind that a featureless AGN continuum would probably fit these data as well. In fact the relative dearth of intermediate-age stellar populations seems to argue against interpreting such blue continua as due solely to O-stars. The short lifetime of such stars $(\sim 3 \mathrm{Myr})$ would imply recurrent and frequent star-formation 

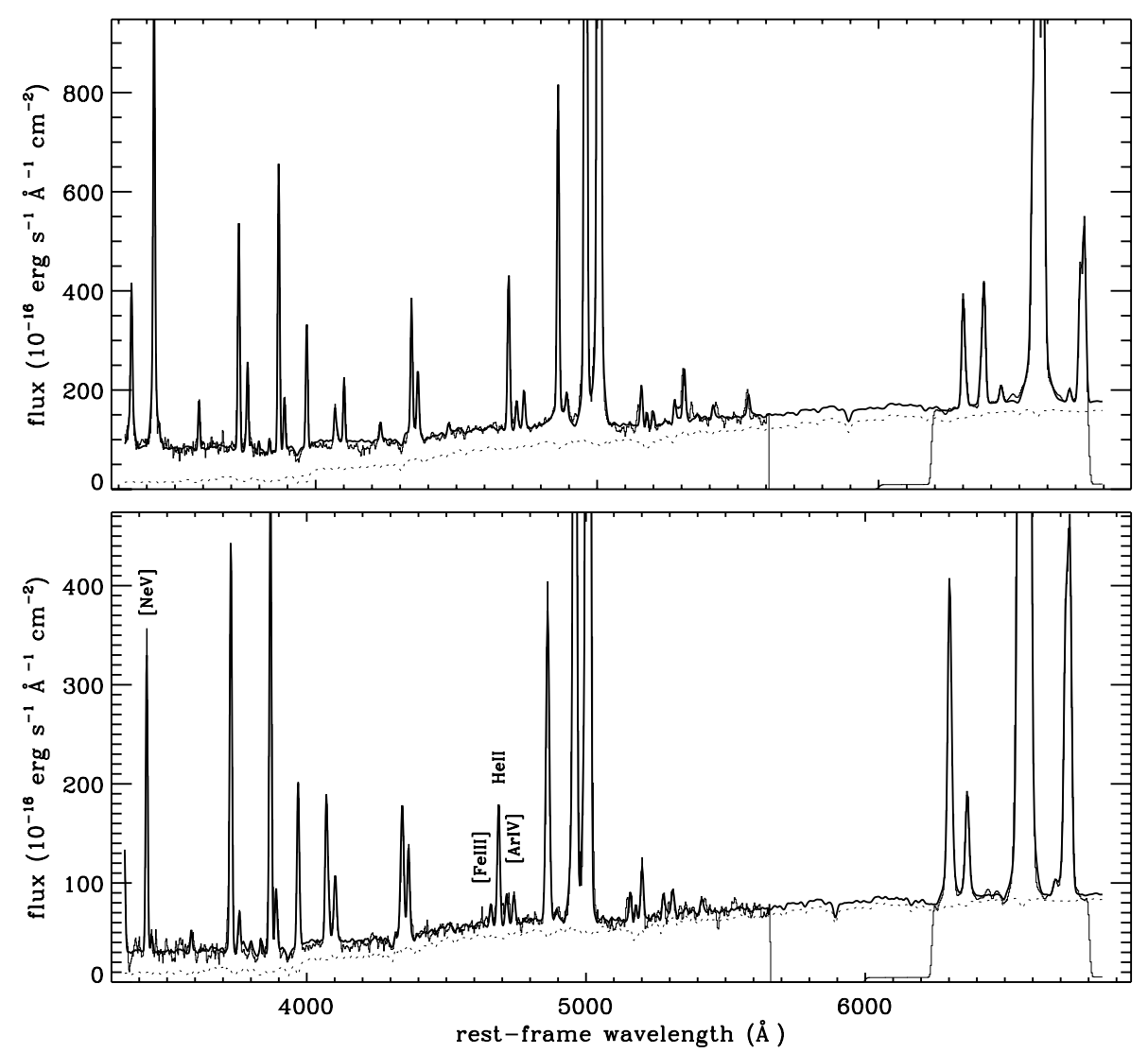

Figure 1. Combined G430L and G750M spectrum for the central $0^{\prime \prime} .25 \times 00^{\prime \prime} .2$ of the Seyfert 2 nuclei in Mrk573 (up) and NGC5427 (down). Overplotted to the data is the best fitting combination of single-age stellar population models from Bruzual \& Charlot (2003) and emission-line Gaussian templates. The dotted line shows the contribution of the oldest, 10-Gyr-old, stellar template. A blue continuum is needed to match the spectrum of these nuclei.

episodes (every $\sim 6 \mathrm{Myr}$ while Seyfert activity lasts) and the build-up of a detectable intermediate-age stellar population if this behaviour have persisted for a 1 Gyr or more. Assuming that galactic nuclei cycle through different kind of active phases and quiescent periods, based on the distribution of host Hubble-types across our sample and on the fraction of Seyfert nuclei in the nearby galaxy population (Ho et al. 1997) our sample nuclei should have spent $\sim 16 \%$ of their recent history shining as Sy2s. Over 1 Gyr they would have thus experienced $\sim 27$ star-formation episodes associated with Seyfert activity, leading to an intermediate-age stellar population that should be detectable. Such populations would indeed have luminosities comparable to that of the blue continua we observe, since for a given mass and for standard initial mass functions, a 1-Gyr-old stellar population is only 60 to 110 times dimmer than a few-Myr-old population.

Furthermore, the luminosity of the blue component needed in our Sy2s correlates well with the strength of the emission from highly-ionised species such as $[\mathrm{Nev}] \lambda 3426$ (Fig. 2), which suggests that the featureless continuum arises from the central engine rather than from star-forming regions. Finally, in the Sy2s requiring a blue continuum, the characteristic $4650 \AA$ "bump" due to Wolf-Rayet stars in strong and metal-rich starbursts is (with one exception) either absent or can also be explained by a superposition of 


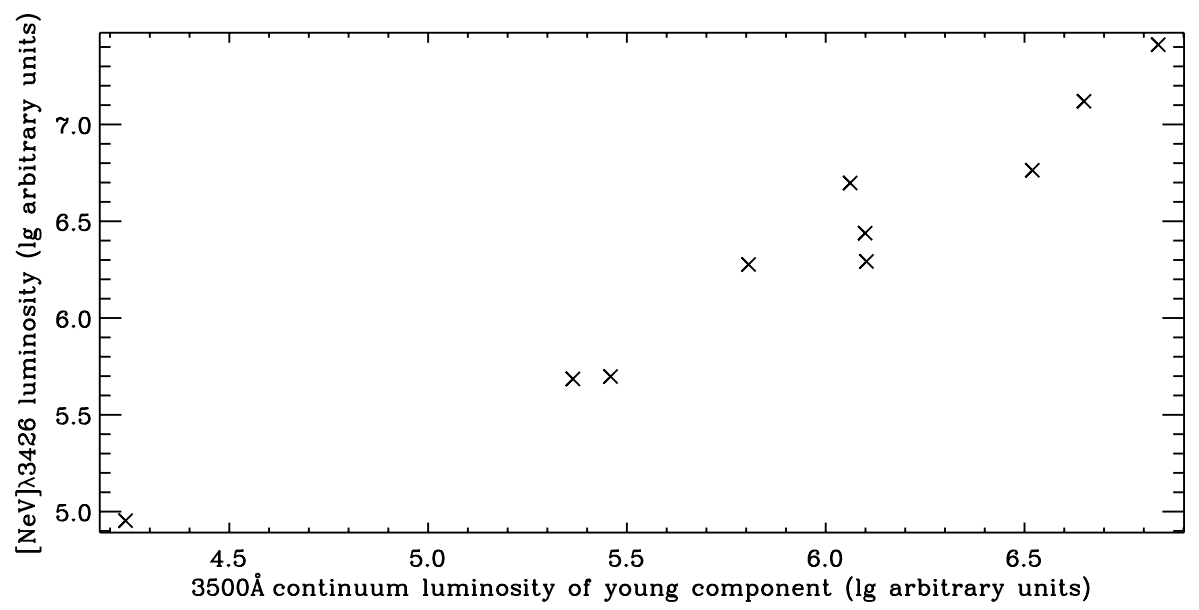

Figure 2. Correlation between the luminosity of the featureless blue continuum, obtained from rescaling the contribution to the observed flux at $3500 \AA$ of the youngest stellar template, and the strength of the $[\mathrm{Nev}] \lambda 3426$ nebular emission in Seyfert 2 nuclei.

forbidden lines such as [ArIv] $\lambda \lambda 4711,4740$, [FeIII] $\lambda 4658$ and [Arv] $\lambda 4625$. Forbidden lines from several other highly-ionised species are also observed in these cases.

Given that the vast majority of our Sy2s do not show evidence of a BLR even when zooming in the central $\sim 30 \mathrm{pc}$, these findings suggest some of our targets could be "true" Type 2 sources, which are predicted to form at low AGN luminosities by some models (e.g., Elitzur \& Shlosman 2006). Alternatively, the small fraction of intermediate-age stellar populations in our sample could be reconciled with on-going star formation in roughly half of our objects if we consider the suggestion of Nayakshin (2006) that only massive stars can form within few pc of central SMBHs. A population characterised by a top-heavy initial mass function would indeed leave little trace of itself after a 1 or 2 Gyr. In this case, however, such star-formation activity must be intimately connected to the central accretion of material, in order to explain the correlation of Fig. 2.

\section{Acknowledgements}

M. Sarzi is grateful to R. Cid-Fernandes for his useful suggestions.

\section{References}

Antonucci, R. 1993,ARAA, 31, 473

Bruzual, G., \& Charlot, S. 2003, MNRAS, 344, 1000

Colina, L., et al. 1997, APJ, 488, L71

González Delgado, R. M., Heckman, T., Leitherer, C., Meurer, G., Krolik, J., Wilson, A. S., Kinney, A., \& Koratkar, A. 1998, APJ, 505, 174

Elitzur, M., \& Shlosman, I. 2006, APJ, 648, L101

Ho, L. C., Filippenko, A. V., \& Sargent, W. L. W. 1997, APJS, 112, 315

Mulchaey, J. S., Regan, M. W., \& Kundu, A. 1997, APJS, 110, 299

Nayakshin, S. 2006, MNRAS, 372, 143

Osterbrock, D. E., \& Martel, A. 1993, APJ, 414, 552

Sarzi, M., Rix, H.-W., Shields, J. C., Ho, L. C., Barth, A. J., Rudnick, G., Filippenko, A. V., \& Sargent, W. L. W. 2005, APJ, 628, 169

Sarzi, M., et al. 2006, MNRAS, 366, 1151

Shields, J. C., et al. 2007, APJ, 654, 125 